\title{
Fatores Associados ao Histórico de Quedas de Idosos Assistidos pelo Programa de Saúde da Família'
}

\section{Factors Associated with the History of Falls of Elderly Assisted by the Family Health Program}

\author{
Natalia Aquaroni Ricci \\ Fisioterapeuta. Mestre em Gerontologia. Doutoranda do Departa- \\ mento de Otorrinolaringologia e Cirurgia de Cabeça e Pescoço da \\ Universidade Federal de São Paulo. \\ Endereço: Rua Pacaembu, 257/ 602, CEP 18040-710, Sorocaba, \\ SP, Brasil. \\ E-mail: natalia_ricciळhotmail.com \\ Daniele de Faria Figueiredo Gonçalves \\ Fisioterapeuta. Mestre em Gerontologia. Professora de Fisioterapia \\ da Universidade Paulista. \\ Endereço: Rua Edmundo Juventino Fuentes, 160/ 91, bloco B, CEP \\ 03280-000, São Paulo, SP, Brasil. \\ E-mail: danieleffœbol.com.br

\section{Ibsen Bellini Coimbra} \\ Médico Reumatologista. Professor Doutor da Faculdade de Ciências \\ Médicas da Universidade Estadual de Campinas. \\ Endereço: Av. Dr. Luiz de Tella, 1033. CEP 13083-000, Campinas, \\ SP, Brasil. \\ E-mail: ibcoimbraœuol.com.br

\section{Arlete Maria Valente Coimbra} \\ Médica Reumatologista. Doutora em Clínica Médica. Programa de \\ Saúde da Família da Universidade Estadual de Campinas. \\ Endereço: Av. Dr. Luiz de Tella, 1033, CEP 13083-000, Campinas, \\ SP, Brasil. \\ E-mail: acoimbra®fcm.unicamp.br \\ I Trata-se de artigo-síntese de Dissertação de Mestrado apresenta- \\ da ao Programa de Pós-Graduação em Gerontologia da Faculdade \\ de Educação da Universidade Estadual de Campinas, por Natalia \\ Aquaroni Ricci, em 2006.
}

\section{Resumo}

Este estudo tem como objetivo identificar os fatores sociodemográficos, clínico-funcionais e psicocognitivos associados ao histórico de quedas de idosos saudáveis. A amostra foi constituída por 96 idosos divididos igualmente em três grupos de acordo com o histórico de quedas ocorrido no último ano (sem queda, uma queda e quedas recorrentes). Não houve diferença entre os grupos quanto aos dados sociodemográficos. Os idosos do grupo sem quedas apresentaram menor queixa de dor $(p=0,012)$ e de tontura ( $\mathrm{p}=\mathrm{o}, \mathrm{oo3})$, melhor mobilidade $(\mathrm{p}<\mathrm{o}, \mathrm{oo1})$, capacidade funcional $(p<0,001)$ e menor relato de quase quedas ( $\mathrm{p}=\mathrm{o}, \mathrm{003}$ ) quando comparados aos idosos dos grupos com histórico de quedas. $\mathrm{Na}$ avaliação psicocognitiva os idosos com quedas recorrentes revelaram maior escore para humor deprimido $(\mathrm{p}=$ o,oog) e maior déficit cognitivo $(\mathrm{p}=\mathrm{o}, \mathrm{O} 4 \mathrm{o})$ do que os sem quedas. As quedas interferem de forma negativa na vida do idoso, motivo da importância de identificação dos fatores protetores e potencializadores deste evento. Com o reconhecimento desses fatores é possível que os profissionais possam prevenir as quedas ou as consequências debilitantes causadas por sua ocorrência.

Palavras-chave: Idoso; Quedas; Programa de saúde da família; Saúde do idoso. 


\section{Abstract}

This study aims at identifying the sociodemographic, clinical-functional and psycho-cognitive factors associated with the history of falls of community-dwelling elderly individuals assisted by Programa Saúde da Família (PSF - Family Health Program). The sample comprised 96 elderly subjects equally divided into three groups according to the report of falls that occurred during the past year, as follows: without falls, one fall or recurrent falls. There were no significant differences between groups in relation to sociodemographic data. The non-faller group presented less complaints about pain ( $p=0.012$ ) and dizziness ( $p=0.003)$, and less near-falls reports $(p=0.003$ ) when compared to the faller groups. Besides, it presented better mobility $(\mathrm{p}<0.001)$ and functional capacity $(\mathrm{p}<0.001)$ in comparison with the same groups. In the psychocognitive assessment, recurrent fallers showed higher depressed mood score $(\mathrm{p}=\mathrm{o.009})$ and higher cognitive impairment score $(\mathrm{p}=\mathrm{0} .040)$ compared to non-fallers. The occurrence of falls must be considered a significant agent that negatively affects elders' life. This is why its causative as well as impeditive factors must be accurately identified, so that professionals can effectively prevent falls or their debilitating consequences.

Keywords: Aged; Falls; Family Health Program; Older adults' health.

\section{Introdução}

A queda é a causa mais comum de acidentes em pessoas com 65 anos ou mais, sendo definida como um evento não intencional que resulta na mudança de posicionamento do indivíduo a um nível inferior ao que se encontrava (Nevitt, 1997). Em estudo populacional brasileiro, com idosos residentes em comunidade, a prevalência de uma queda no período de um ano foi de $30,9 \%$ e de quedas recorrentes foi 10,8\% (Perracini e Ramos, 2002).

Dados provenientes do Ministério da Saúde do Brasil revelam que, entre os idosos, acima de 60 anos, as quedas ocupam o terceiro lugar na mortalidade por causas externas e em relação à morbidade, são responsáveis pelo primeiro lugar $(56,1 \%)$ das internações (Gawryszewski e col., 2004). Entretanto, independentemente da gravidade da lesão, o impacto psicológico negativo causado por uma queda tem sequelas devastadoras para a vida do idoso (Chandler, 2002). O que ocorre é um ciclo que se inicia com a queda e tem como consequência primária o medo de cair (Rekeneire e col., 2003). Esse medo resulta na perda da confiança na realização das tarefas do cotidiano, restrição nas atividades sociais e dependência aumentada que, por sua vez, levam ao descondicionamento (Boulgarides e col., 2003), restrição da mobilidade e isolamento social. O ciclo se conclui, finalmente, com a perda da capacidade funcional (Chandler, 2002) e, por conseguinte, em maior suscetibilidade do idoso a sofrer novas quedas.

Dessa forma, a elevada incidência das quedas na população idosa e suas consequências acarretam altos custos para o sistema de saúde e interferem diretamente na qualidade de vida desses indivíduos (Boulgarides e col., 2003). Embora se reconheça a necessidade de instaurar medidas preventivas e de tratamento com a finalidade de solucionar os problemas em nível social e individual acarretados pelas quedas, há dificuldade em reconhecer o motivo da ocorrência desse evento, pois as quedas têm origem multifatorial, não sendo possível isolar um único fator como determinante para seu acontecimento.

Alguns fatores já são reconhecidos como associados às quedas, porém diferem em relação ao histórico pregresso de quedas do indivíduo e, também, quanto à população idosa estudada (comunidade, institucio- 
nalizada e hospitalizada). Além disso, o risco para quedas aumenta linearmente com o aumento no número de fatores associados (Nevitt, 1997).

Quanto ao histórico, as quedas recorrentes são frequentemente atribuídas a fatores intrínsecos, enquanto quedas esporádicas têm maior associação com fatores ambientais e diminuição da atenção (Morris e col., 2004). Para a população idosa em geral, os principais fatores de risco intrínsecos são: a idade avançada, sexo feminino, incapacidade funcional, déficit de equilíbrio, distúrbios de marcha, baixa aptidão física, diminuição da força muscular, hipotensão postural, baixa acuidade visual, déficits cognitivos e polifarmácia (Nevitt, 1997).

A identificação dos fatores associados às quedas específicos aos idosos é dificultada pela inclusão nas amostras de indivíduos com diferentes graus de dependência (Rekeneire e col., 2003).

Entre a população idosa, cresceu o número daqueles que se beneficiaram da assistência à saúde oferecida pelo PSF. Atualmente, o PSF tem contribuído para que estudos sejam realizados, a fim de identificar os problemas de saúde e situações de risco aos quais a comunidade está exposta (Brasil, 2004). Com o conhecimento dos fatores que possam trazer prejuízos à saúde do idoso, a assistência na estratégia do PSF pode desenvolver programas de promoção, proteção e recuperação da saúde, de forma integral e contínua, inclusive aqueles voltados diretamente à problemática das quedas nessa população.

Devido ao caráter debilitante das quedas, para compreender e possibilitar a redução na frequência e nos danos causados por elas, essa pesquisa tem como objetivo identificar os fatores sociodemográficos, clínico-funcionais e psicocognitivos associados ao histórico de quedas de idosos.

\section{Método}

Foi realizada pesquisa quantitativa, por meio de estudo descritivo, comparativo de corte transversal. A pesquisa foi previamente aprovada pelo Comitê de Ética em Pesquisa da Faculdade de Ciências Médicas da Universidade Estadual de Campinas (protocolo $n^{0} 766 / 2005$ ).

A amostra, deste estudo é proveniente de pesquisa populacional sobre o envelhecimento e foi constituída por idosos residentes em uma comunidade atendida pelo PSF, no município de Amparo, Estado de São Paulo (Coimbra e col., 2010). O banco de dados do levantamento populacional foi consultado, a fim de selecionar, segundo critérios de inclusão e exclusão, os participantes deste estudo. Os critérios de inclusão foram: idade igual ou superior a 65 anos, ambos os sexos e deambulação sem dispositivos auxiliares para a marcha. Foram excluídos os idosos que apresentavam: incapacidade de compreender e atender a comando verbal simples; amputações e/ou uso de próteses em membros; sequelas de acidente vascular encefálico; doença de Parkinson; fraturas em membros inferiores e/ou coluna após os 65 anos; doença coronariana grave; labirintopatia incapacitante; neuropatia diagnosticada e deformidade nos pés.

Os idosos que atenderam aos critérios de elegibilidade foram divididos quanto ao histórico de quedas no último ano, de forma a compor três grupos (Grupo 1: sem quedas, Grupo 2: uma queda e Grupo 3: quedas recorrentes). A divisão pelo grupo pertencente foi realizada em consulta ao banco de dados populacional, que possuía a informação do número de quedas por ano de cada indivíduo. Contudo, houve necessidade de realizar contatos telefônicos prévios para verificar possíveis mudanças quanto ao histórico de quedas entre o intervalo de tempo (de quatro a oito meses) da avaliação do projeto populacional e a da presente pesquisa, possibilitando o reagrupamento de alguns casos.

Os grupos foram compostos de 32 sujeitos cada, perfazendo 96 idosos na amostra total. Para redução do número de sujeitos foi realizada escolha dos casos por filtro de homogeneização quanto ao sexo e faixa etária (65-69 anos, 70-74 anos, 75-79 anos e 80 anos e mais).

A seleção dos sujeitos pelo banco do projeto populacional até perfazer a amostra da presente pesquisa segue esquematizada na figura 1.

Todos os grupos foram submetidos ao mesmo protocolo de pesquisa, que incluiu as seguintes avaliações: questionário sociodemográfico, avaliação clínico-funcional e avaliação psicocognitiva. 0 banco de dados do levantamento populacional não foi consultado para a coleta das informações referentes ao protocolo desta pesquisa.

Os dados sociodemográficos foram obtidos por meio de questionário estruturado em forma de en- 
Figura I - Esquematização do processo de seleção dos idosos

$\begin{aligned} & \text { Consulta pela } \\
& \text { pesquisadora principal }\end{aligned} \quad \begin{aligned} & \text { Critérios de inclusão/ exclusão } \\
& \text { Unidades de Saúde }\end{aligned} \mid$\begin{tabular}{l|l} 
Consulta sobre quedas & Filtro por sexo e idade
\end{tabular}

Banco de dados do levantamento populacional de idosos do município de Amparo
Excluidos

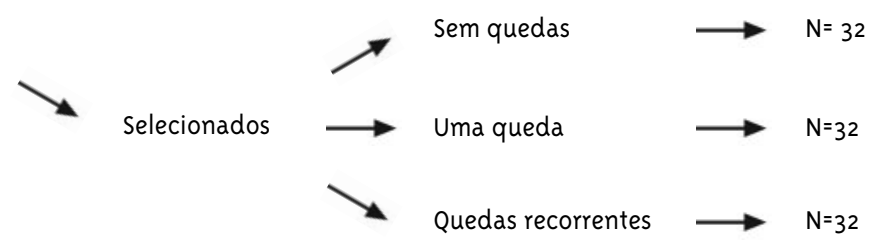

trevista, no qual constavam informações sobre sexo, idade, cor (autorreferida), estado conjugal (com ou sem parceiro), grau de escolaridade e arranjo de moradia (sozinho, uma geração (marido/filhos), duas gerações (filhos/netos)).

Os dados clínico-funcionais avaliados foram: número de doenças e medicamentos, queixa de tontura e dor musculoesquelética, estado nutricional, força muscular, realização de atividade física, acuidade visual, capacidade funcional, mobilidade e dados sobre quedas.

O número de hipóteses diagnósticas e medicamentos foram coletados do prontuário do paciente, referentes à última consulta no PSF. Os relatos de queixa de tontura e de dor musculoesquelética foram referentes aos sintomas persistentes nos últimos seis meses, que caracteriza a cronicidade da sintomatologia.

A atividade física foi considerada regularmente praticada, pela participação do idoso em atividades físicas no tempo livre por três ou mais vezes na semana por mais de trinta minutos nas últimas duas semanas, de acordo com a definição de Yusuf e colaboradores (1996).

A avaliação da acuidade visual foi realizada por meio da tabela de Snellen. Como na presente pesquisa a intenção era verificar a funcionalidade da visão, foi permitido usar lentes corretivas, caso ela fosse de uso habitual, e os dois olhos foram avaliados simultaneamente. A classificação da acuidade visual foi feita seguindo as recomendações do CID-9 (World Healt Organization, 1977) em visão normal $(0,7)$, próxima do normal (o,6 a o,3), baixa visão moderada $(0,25$ a 0,12$)$ e baixa visão grave $(0,1)$.

Os dados antropométricos de peso (quilogramas - kg) e altura (metros - m) foram aferidos por balança de consultório, marca Filizola ${ }^{\circledR}$, e com valores foi calculado o Índice de Massa Corpórea (IMC). 0 estado nutricional foi classificado conforme o nível de corte do IMC, específico para a população idosa, em desnutrição $(<22,0)$, eutrofismo $(22,0<27,0)$ e obesidade (27,0) (Lipschitz, 1994).

A avaliação muscular foi aferida pela força de preensão palmar, por meio de dinamômetro manual, marca Grip Track ${ }^{T M}$ Commander. Foi considerada a média em kg de três tentativas com a mão dominante do paciente. 0 teste foi realizado com o indivíduo sentado com o braço aduzido e paralelo ao tronco, cotovelo fletido a 90 graus, antebraço e punho em posição neutra.

Para a avaliação da capacidade funcional foi aplicado o instrumento validado transcultural para o Brasil, Brazilian OARS Multidimensional Functional Assessment Questionnaire (BOMFAQ) (Ramos e col., 1993). 0 instrumento é composto de 15 atividades, sobre as quais o idoso responde sobre a sua capacidade atual de realizá-las classificandoas em "sem dificuldade", "pouca dificuldade" ou "muita dificuldade". O escore total de atividades comprometidas foi feito pela soma das respostas das categorias "pouca" e "muita" dificuldade, sendo que quanto maior a pontuação maior o prejuízo na capacidade funcional.

A mobilidade foi avaliada pelo Time Up and Go 
Test (TUGT) (Podsiadlo e Richardson, 1991). Esse instrumento foi criado com o objetivo principal de analisar o equilíbrio, risco de quedas e mobilidade de idosos (Podsiadlo e Richardson, 1991). O teste consiste na cronometragem do tempo despendido pelo indivíduo no percurso de levantar-se de uma cadeira, caminhar três metros em linha reta, retornar à cadeira e sentar-se.

A tendência a quedas foi definida como recuperação do equilíbrio anteriormente ao contato com nível inferior e o medo de sofrer quedas foi relatado como presente ou ausente.

A avaliação psicocognitiva foi realizada em entrevista a responder os instrumentos Mini Exame do Estado Mental (MEEM) (Lourenço e Veras, 2006) e o Geriatric Depression Scale (GDS) (Paradela e col., 2005), ambos os testes validados e traduzidos transculturalmente para o português do Brasil. O MEEM é um instrumento de rastreio cognitivo, pontuações inferiores a 24 pontos de um total de 30 são consideradas como indício de déficit cognitivo (Lourenço e Veras, 2006). O GDS é um teste de saúde mental, sua versão reduzida tem 15 questões referentes ao estado emocional do idoso na semana anterior à avaliação, com respostas do tipo sim/ não, sendo os valores abaixo de seis pontos considerados como normalidade e acima indicativo de humor deprimido (Paradela e col., 2005).

Os idosos elegíveis para a pesquisa foram convidados para a avaliação e aqueles que se dispuseram a participar voluntariamente do estudo assinaram o Termo de Consentimento Livre e Esclarecido, conforme preconizado pela Resolução 196/96 do Conselho Nacional de Saúde. Os participantes inicialmente foram convocados pelo pesquisador independente, que tinha conhecimento do grupo ao qual o idoso pertencia, a comparecer a UBS para a aplicação do protocolo de avaliação. 0 teste de equilíbrio foi realizado por “pesquisador cego". Para a realização padronizada do protocolo de avaliação os examinadores envolvidos na pesquisa passaram por treinamento prévio para a aplicação dos testes.

Para a caracterização da amostra por grupos foi realizada análise descritiva simples dos dados. A análise inferencial da comparação entre os grupos quanto às variáveis qualitativas categóricas foi feita pelo teste Qui-quadrado $\left(\chi^{2}\right)$. Na ocorrência de diferença significativa entre os grupos $(p<0,05)$ para o Qui-quadrado, foi aplicada partição da tabela para estabelecer a comparação múltipla e verificar os pares de diferença. $\mathrm{Na}$ análise comparativa das variáveis quantitativas entre os grupos foi utilizado o Teste de Kruskal-Wallis devido à ausência de distribuição normal. Para as variáveis cujo valor do Kruskal-Wallis foi significante ( $\mathrm{p}<\mathrm{o}, 05$ ), utilizou-se o teste de Mann-Whitney para análise de comparação entre os pares de grupos.

O nível de significância adotado para os testes estatísticos foi de $5 \%(\alpha=0,05)$. As análises estatísticas foram realizadas pelo programa computacional Statistical Package for the Social Sciences- SPSS, versão 10.0.

\section{Resultados}

A amostra total foi constituída por 96 idosos da comunidade atendidos pelo PSF, distribuídos igualmente, quanto ao sexo (50,0\% masculino e 50,0\% feminino) e faixa etária ( $25 \%$ de casos por faixa etária), em três grupos ( $\mathrm{n}=32$ idosos) referentes ao histórico de quedas.

Em conseqüência da divisão por faixa etária, as médias da idade, em anos, não apresentaram diferenças estatísticas ( $p=0,968)$ entre o Grupo 1 (74,81 7,25 anos), Grupo 2 (75,19 7,32 anos) e Grupo 3 (74,47 6,39 anos).

A amostra total, assim como por grupos, foi composta de maioria branca $(87,5 \%)$, com vida conjugal $(66,7 \%)$, com nível de escolaridade de primário completo $(42,7 \%)$ e com arranjo de moradia de uma geração $(75,0 \%)$. Os dados sociodemográficos dos grupos estão detalhados na Tabela 1.

Os idosos com diferentes históricos de quedas, representados pelos grupos, não apresentaram diferenças significantes quanto aos dados sociodemográficos analisados. Quanto às frequências, embora sem diferença significativa, o Grupo 3 obteve maior número de casos sem vida conjugal, analfabetismo e arranjo de moradia só, quando comparado com os demais grupos.

A Tabela 2 mostra as frequências dos dados clínicofuncionais dos grupos de idosos quanto às quedas.

No Grupo 1, a maioria dos idosos (59,4\%) apresentou uma ou duas doenças associadas, enquanto nos grupos que sofreram quedas, a maioria ( $50 \%$ ) tinha três ou quatro doenças concomitantes. Nos três 
Tabela I - Análise comparativa da caracterização sociodemográfica de 96 idosos saudáveis distribuídos por grupos de histórico de quedas. Amparo-SP, 2006

\begin{tabular}{|c|c|c|c|c|c|}
\hline & \multirow[t]{2}{*}{ Categorias } & \multicolumn{3}{|c|}{ Frequência n (\%) } & \multirow{2}{*}{$\begin{array}{l}\text { Qui-Quadrado } \\
\text { p-valor* }\end{array}$} \\
\hline & & Grupo I & Grupo 2 & Grupo 3 & \\
\hline Cor & $\begin{array}{l}\text { Branca } \\
\text { Negra } \\
\text { Parda }\end{array}$ & $\begin{array}{c}30(93,8) \\
1 \quad(3,1) \\
1 \quad(3,1)\end{array}$ & $\begin{array}{ll}27 & (84,4) \\
3 & (9,4) \\
2 & (6,2)\end{array}$ & $\begin{array}{l}27(84,4) \\
4(12,5) \\
1 \quad(3,1)\end{array}$ & 0,651 \\
\hline Estado conjugal & $\begin{array}{l}\text { Com parceiro } \\
\text { sem parceiro }\end{array}$ & $\begin{array}{l}25(78,1) \\
7(21,9)\end{array}$ & $\begin{array}{l}20(62,5) \\
12(37,5)\end{array}$ & $\begin{array}{l}19(59,4) \\
13(40,6)\end{array}$ & 0,234 \\
\hline $\begin{array}{l}\text { Grau de } \\
\text { escolaridade }\end{array}$ & $\begin{array}{l}\text { Analfabeto } \\
\text { Primário incompleto } \\
\text { Primário completo } \\
\text { Ensino Médio completo }\end{array}$ & $\begin{array}{l}2(6,2) \\
10(31,3) \\
13(40,6) \\
7(21,9)\end{array}$ & $\begin{array}{c}6(18,8) \\
6(18,8) \\
16(50,0) \\
4(12,4)\end{array}$ & $\begin{array}{c}10(31,2) 0,176 \\
7(21,9) \\
12(37,5) \\
3(9,4)\end{array}$ & \\
\hline $\begin{array}{l}\text { Arranjo de } \\
\text { moradia }\end{array}$ & $\begin{array}{l}\text { Só } \\
\text { Com I geração } \\
\text { Com } 2 \text { gerações }\end{array}$ & $\begin{array}{l}3(9,4) \\
21(65,6) \\
8(25,0)\end{array}$ & $\begin{array}{c}1(3,1) \\
29(90,6) \\
2(6,3)\end{array}$ & $\begin{array}{c}5(15,6) \\
22(68,8) \\
5(15,6)\end{array}$ & 0,097 \\
\hline
\end{tabular}

${ }^{*}$ Nível de significância $=0,05$

grupos, a maioria dos idosos fazia uso de três ou quatro medicamentos (40,6\%). Na comparação entre os grupos não foi verificada diferença estatística para as doenças e medicamentos associados.

A amostra total e a divisão por grupos revelaram que a maioria dos idosos não praticava atividade física (76\%). 0 diagnóstico nutricional pelo IMC demonstrou que a amostra total foi constituída por uma maioria de indivíduos obesos (50\%), contudo, no Grupo 1 a maior prevalência foi de sujeitos eutróficos (50\%). Os participantes do estudo apresentaram acuidade visual próxima ao normal (41,7\%), enquanto nos Grupo 1 e 3 a maior prevalência foi de casos de visão normal, porém sem diferença significativa entre as frequências dos grupos. Para essas variáveis clínico-funcionais não foram encontradas diferenças estatísticas entre os grupos.

Quanto à dor, 61,5\% dos idosos referiam sentir dores pelo corpo, no entanto, o Grupo 1 teve o maior percentual sem essa queixa $(62,5 \%)$. O relato de dor teve diferença significativa entre os grupos $(p=0,012)$ que na partição da tabela foi identificada entre o Grupo 1 e 2 ( $p=0$,oo5) e Grupo 1 e 3 ( $p=$ $0,012)$. A maioria da amostra não referiu queixa de tontura $(64,5 \%)$, porém essa foi estatisticamente diferente entre os grupos $(\mathrm{p}=0,003)$ e pela partição foi revelada entre o Grupo 1 e 2 ( $p=0,051)$ e Grupo 1 e $3(p=0,007)$. A análise comparativa mostrou que as queixas de dor e tontura são mais prevalentes entre os idosos que sofreram quedas do que entre os que não sofreram.

A maioria dos idosos dos três grupos relatou sentir medo de cair; quanto à quase quedas, o Grupo 3 obteve maioria de casos afirmativos (56,3\%). Os grupos apresentaram diferença estatística para quase quedas $(p=0,003)$, sendo pela partição da tabela encontrada entre os Grupos 1 e 3 (p= o,o11) e Grupos 1 e 2 ( $p$ = o,o23).

A variabilidade e comparação da força de preensão palmar, do BOMFAQ e TUGT dos grupos estão apresentadas na Tabela 3 .

Os grupos não apresentaram diferenças significantes quanto à força de preensão.

$\mathrm{Na}$ avaliação funcional, as médias obtidas pelo BOMFAQ revelaram um aumento na dificuldade de realização das atividades, conforme a maior ocorrência do evento quedas pelos grupos. Os grupos apresentaram diferença significativa no BOMFAQ ( $\mathrm{p}=\mathrm{o}, \mathrm{oo1})$, a qual foi encontrada entre o Grupo 1 e 2 ( $p$ = o,o32) e Grupo 1 e 3 ( $p<$ o,oo1). Pela análise comparativa, os idosos sem quedas obtiveram melhor funcionalidade pelo BOMFAQ do que os grupos com quedas.

O tempo despendido no TUGT também apresentou diferença estatística entre os grupos $(\mathrm{p}=\mathrm{o}, \mathrm{OO} 2)$, 
Tabela 2 - Análise comparativa dos dados clínico-funcionais de 96 idosos saudáveis distribuídos por grupos de histórico de quedas. Amparo-SP, 2006

\begin{tabular}{|c|c|c|c|c|c|}
\hline & \multirow{2}{*}{ Categorias } & \multicolumn{3}{|c|}{ Frequência n (\%) } & \multirow{2}{*}{$\begin{array}{l}\text { Qui-Quadrado } \\
\text { p-valor* }\end{array}$} \\
\hline & & Grupo I & Grupo 2 & Grupo 3 & \\
\hline \multirow{4}{*}{$\begin{array}{l}\text { Número de doenças } \\
\text { associadas }\end{array}$} & Sem doença & - & - & $1(3,1)$ & \\
\hline & 1 ou 2 doenças & $19(59,4)$ & $9(28,1)$ & $10(31,3)$ & 0,122 \\
\hline & 3 ou 4 doenças & $9(28,1)$ & $16(50,0)$ & $16(50,0)$ & \\
\hline & 5 ou mais doenças & $4(12,5)$ & $7(21,9)$ & $5(15,6)$ & \\
\hline \multirow{4}{*}{$\begin{array}{l}\text { Número de } \\
\text { medicamentos }\end{array}$} & Não faz uso & $1(3,1)$ & - & $2(6,3)$ & \\
\hline & I ou 2 medicamentos & $13(40,6)$ & $11(34,4)$ & $9(28,1)$ & 0,733 \\
\hline & 3 ou 4 medicamentos & $13(40,6)$ & $13(40,6)$ & $13(40,6)$ & \\
\hline & 5 ou mais medicamentos & $5(15,7)$ & $8(25,0)$ & $8(25,0)$ & \\
\hline \multirow[t]{3}{*}{ Estado nutricional } & Desnutrição & $2(6,2)$ & $4(12,5)$ & $3(9,4)$ & \\
\hline & Eutrofismo & $16(50,0)$ & $11(34,4)$ & $12(37,5)$ & 0,714 \\
\hline & Obesidade & $14(43,8)$ & $17(53,1)$ & $17(53,1)$ & \\
\hline \multirow[t]{3}{*}{ Acuidade visual } & Normal & $15(46,9)$ & $11(34,4)$ & $13(40,7)$ & \\
\hline & Próxima do normal & $14(43,8)$ & $14(43,8)$ & $12(37,5)$ & 0,610 \\
\hline & Baixa visão moderada & $3(9,3)$ & $7(19,8)$ & $7(19,8)$ & \\
\hline \multirow[t]{2}{*}{ Dor } & Sim & $12(37,5)$ & $24(75,0)$ & $23(71,9)$ & 0,003 \\
\hline & Não & $20(62,5)$ & $8(25,0)$ & $9(28,1)$ & \\
\hline \multirow[t]{2}{*}{ Tontura } & sim & $5(15,6)$ & $13(40,6)$ & $16(50,0)$ & 0,012 \\
\hline & Não & $27(84,4)$ & $19(59,4)$ & $16(50,0)$ & \\
\hline \multirow[t]{2}{*}{ Atividade física } & Sim & $11(34,4)$ & $7(21,9)$ & $5(15,6)$ & 0,202 \\
\hline & Não & $21(65,6)$ & $25(78,1)$ & $27(84,4)$ & \\
\hline \multirow[t]{2}{*}{ Medo de quedas } & $\operatorname{sim}$ & $16(50,0)$ & $21(65,6)$ & $20(62,5)$ & 0,404 \\
\hline & Não & $16(50,0)$ & $11(34,4)$ & $12(37,5)$ & \\
\hline \multirow[t]{2}{*}{ Quase quedas } & $\operatorname{sim}$ & $5(15,6)$ & $14(43,8)$ & $18(56,2)$ & 0,003 \\
\hline & Não & $27(84,4)$ & $18(56,2)$ & $14(43,8)$ & \\
\hline
\end{tabular}

* Nivel de significância $=0,05$

sendo encontrada nos Grupos 1 e 2 ( $\mathrm{p}$ = o,oo1) e Grupo 1 e 3 ( $\mathrm{p}=\mathrm{o}, \mathrm{oo1})$. Os grupos com quedas apresentaram pior desempenho na mobilidade do que os idosos sem quedas.

Na avaliação psicocognitiva a maioria dos idosos dos Grupos 1 e 2 apresentou normalidade para a identificação de problemas tanto no MEEM como no GDS. O Grupo 3 obteve maior frequência de casos para o $G D S$, que sugere humor deprimido, e para o MEEM déficit cognitivo. As frequências e comparação dos grupos quanto aos dados psicocognitivos estão expostos na Tabela 4.
O GDS apresentou diferença significativa entre os grupos $(\mathrm{p}=\mathrm{o}, 014)$ e pela partição da tabela foi revelada entre o Grupo 1 e 3 ( $p=$ o,oog) e Grupo 2 e 3 ( $p=0,021)$, demonstrando que os idosos com quedas recorrentes apresentam maior escore para humor deprimido conforme a pontuação no $G D S$ do que os demais. O MEEM revelou tendência à diferença estatística entre os grupos e quando analisado pelos pares de grupos foi verificada diferença significativa entre o Grupo 1 e 3 ( $p=$ o,o40), isto é, maior declínio cognitivo pelo escore do MEEM em idosos com quedas recorrentes em relação aos sem quedas. 
Tabela 3 - Análise comparativa da variabilidade da força de preensão e do Brazilian OARS Multidimensional Functional Assessment Questionnaire de 96 idosos saudáveis distribuídos por grupos de histórico de quedas. Amparo-SP, 2006.

\begin{tabular}{llllccc} 
& Crupos & Média & DP & Valor mínimo & Valor máximo & Kruskal-Wallis p-valor * \\
Força de & Grupo I & 29,34 & 9,31 & 13,0 & 49,0 & \\
preensão & Grupo 2 & 25,73 & 9,81 & 13,0 & 49,0 & 0,206 \\
& Grupo 3 & 27,31 & 8,86 & 14,0 & 44,0 & \\
BOMFAQ & Grupo I & 1,50 & 1,70 & 0 & 6 & \\
& Grupo 2 & 3,34 & 3,29 & 0 & 10 & $<0,001$ \\
& Grupo 3 & 3,94 & 2,61 & 0 & 11 & \\
TUGT & Grupo 1 & 11,43 & 2,95 & 7,60 & 20,00 & $<0,001$ \\
& Grupo 2 & 14,57 & 4,23 & 9,30 & 25,00 & \\
& Grupo 3 & 14,48 & 4,46 & 8,10 & 28,00 & \\
\hline
\end{tabular}

* Nível de significância $=0,05$

Tabela 4 - Análise comparativa dos dados psicocognitivos de 96 idosos saudáveis distribuídos por grupos de histórico de quedas. Amparo-SP, 2006.

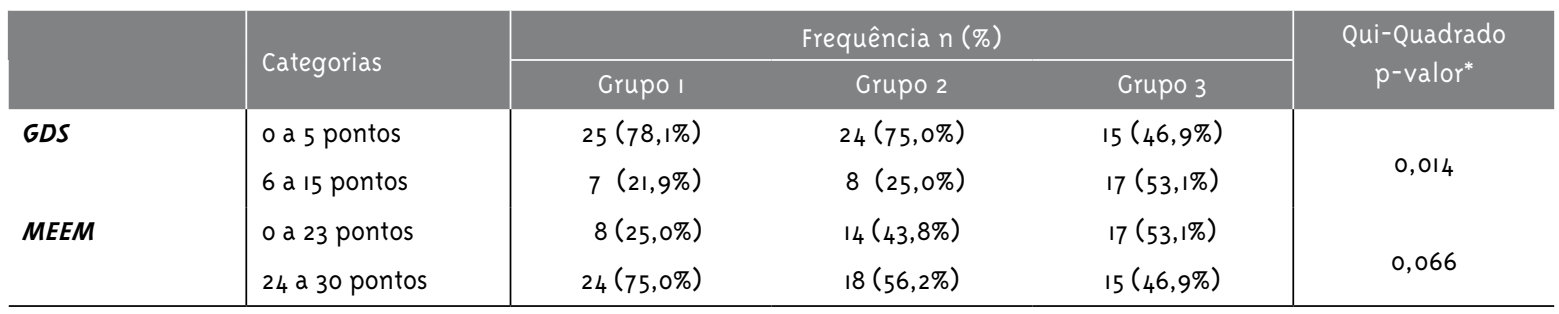

*Nível de significância $=0,05$.

\section{Discussão}

O reconhecimento de fatores associados a não ocorrência, eventual e múltiplas quedas, possibilita o desenvolvimento de estratégias de tratamento para essas três situações. A identificação desses fatores nos idosos da comunidade deve ser feita por meio de uma avaliação global, pois como visto suas causas podem ser as mais variadas.

A classificação das quedas em esporádicas e recorrentes é uma medida adotada nesta pesquisa com o intuito de melhor avaliar os idosos, visto que os mecanismos e fatores de risco para a ocorrência desses eventos diferem (Nevitt, 1997). Os grupos foram divididos igualmente em sexo e faixa etária para que a comparação dos dados quanto ao histórico de quedas fosse isenta da influência desses fatores. Os dados de caracterização sociodemográfica da amostra total, assim como dos grupos, são compatíveis com estudos populacionais brasileiros sobre idosos (Ramos e col., 1998; Lebrão e Duarte, 2003). Os dados sociodemográficos estiveram associados às quedas em uma coorte de 1.415 idosos da cidade de São Paulo (Perracini e Ramos, 2002), no qual a maioria dos idosos "caidores" era analfabeta, não tinha vida conjugal e morava só. Esses dados foram encontrados com maior frequência no grupo de quedas recorrentes, porém sem diferença significativa com os demais. Assim, torna-se improvável que o evento quedas seja atribuível ao status sociodemográfico neste estudo.

Em relação às doenças associadas, em estudo populacional brasileiro com idosos da comunidade foi encontrada maior prevalência de casos com cinco ou mais doenças (33,2\%) (Siqueira e col., 2007), resultado que difere da amostra total deste estudo, 
no qual a maioria dos idosos tinha de três doenças a quatro. Vale ressaltar que foram excluídos desta pesquisa os idosos com doenças limitantes, os quais geralmente possuem diversas comorbidades, motivo pelo qual pode ter ocorrido esta diferença.

O número elevado de comorbidades é com frequência maior em "caidores" quando comparados com idosos sem histórico de quedas (Rekeneire e col., 2003; Morris e col., 2004). Morris e colaboradores (2004) verificaram que idosos com três ou mais doenças associadas têm maior chance de cair ocasionalmente e múltiplas vezes. Embora não tenha havido diferença entre os grupos quanto às doenças associadas, aqueles com histórico de quedas, seguindo as estatísticas dos estudos supracitados, apresentaram maior prevalência de casos (50\%) na categoria "três ou quatro doenças", enquanto a maioria dos idosos sem quedas $(59,4 \%)$ tinha de "uma ou duas doenças".

A maioria dos idosos usava três ou quatro medicamentos, valores superiores aos encontrados em amostra de idosos atendidos em UBS, na qual 39,1\% utilizavam um ou dois medicamentos (Siqueira e col., 2007). O número de medicamentos utilizados é um forte preditor para quedas (Nevitt, 1997) e, embora não tenham ocorrido diferenças entre os grupos, a alta prevalência de medicação nos idosos deste estudo, pode evidenciar necessidade de medidas preventivas quanto à associação da polifarmácia e o risco de futuras quedas, sendo essencial, ao prescrever medicamentos, que se estabeleça uma avaliação criteriosa sobre a precisão de uso e o ajuste de dosagem (Fabrício e col., 2004).

A visão para longe avaliada de forma objetiva revelou não haver diferenças na acuidade visual entre os grupos. A maioria dos idosos (40,6\%) da amostra tinha acuidade visual normal, isto é, acima de o,7 na tabela de Snellen, resultados semelhantes ao estudo de Romani (2005) com idosos longevos, que concluiu que essa normalidade visual pode ser indicativa de boa condição física geral e qualidade de vida. No entanto, vale ressaltar que assim como nesta pesquisa, as medidas visuais objetivas não foram detectadas como diferentes entre caidores e não caidores em outro estudo (Stalenhoef e col., 2002), enquanto a avaliação subjetiva pela autopercepção da condição visual em ruim ou péssima esteve associada às quedas ocasionais e recorrentes (Morris e col., 2004; Perracini e Ramos, 2002).

O diagnóstico nutricional revelou uma maioria obesa na amostra total (50,0\%), ao contrário do que se encontra na literatura, que identifica como fator associado às quedas o baixo índice de massa corpórea (Herdon e col., 1997). O aumento no peso corporal e o acúmulo de gordura nos idosos parecem resultar de uma modificação genética, de mudanças na dieta e nível de atividade física ou a interação desses fatores com o envelhecimento (Matsudo e col., 200o). Medidas preventivas podem ser adotadas a fim de diminuir o IMC dessa população e com isso melhorar a condição de saúde e estabilizar doenças em curso.

A força de preensão palmar identificada como fator de forte evidência para risco de quedas e com menores valores significantes para caidores (Nevitt, 1997; Stalenhoef e col., 2002) não apresentou, no entanto, diferença nos grupos, não podendo estar associada às quedas nessa população. Valores de preensão palmar abaixo de $22 \mathrm{~kg}$ para homens e 12 $\mathrm{kg}$ para mulheres foram considerados como risco para quedas recorrentes em idosos da comunidade (Stalenhoef e col., 2002), a média do grupo de quedas recorrentes foi de $27,31 \mathrm{~kg}$, não estando dentro dos parâmetros para população de risco.

A tontura é uma queixa comum na população idosa, pois com o envelhecimento o sistema vestibular apresenta um decréscimo funcional. Mesmo não tendo maior prevalência na amostra total $(35,5 \%)$, a queixa de tontura teve alto relato e foi ainda maior nos grupos com quedas. No estudo de Tinetti e colaboradores (2000) $24 \%$ de 1.087 idosos da comunidade reportaram esse tipo de sintoma, e a tontura crônica esteve associada à ocorrência de quedas. Esses dados corroboram os resultados deste estudo e, assim, é possível inferir que a ocorrência de tonturas entre os idosos dos Grupos 2 e 3 tenha sido um dos fatores contribuintes ao evento quedas.

0 relato de dor é comum na população idosa e a dor de origem musculoesquelética tem destaque nesse tipo de queixa (Brochet e col., 1998). A dor pode desencadear posturas antálgicas e claudicações que, por sua vez, alteram a postura, o equilíbrio e a marcha, tornando-se um fator de risco para quedas, motivo pelo qual possivelmente a maioria dos idosos dos grupos com quedas apresentaram queixa, sendo essa significativamente superior em relação ao gru- 
po sem quedas, achados semelhantes ao estudo de Rekeneire e colaboradores (2003). A queixa de dor musculoesquelética dos Grupos 2 e 3 foi superior (acima de 70\%) à encontrada no estudo populacional de Brochet e colaboradores (1998), em que $41 \%$ de 741 idosos relataram dor. Esses dados revelam que a presença de dor pode ter sido um dos fatores causadores das quedas e que sua alta prevalência requer medidas de reabilitação, já que a dor tem entre suas consequências a limitação funcional e o impacto negativo no desempenho das atividades diárias.

A maioria dos idosos da amostra total (76\%) não praticava atividade física regularmente, sendo que esta frequência aumentou conforme o histórico de quedas. Em pesquisa populacional com idosos (Yusuf e col., 1996), foram encontradas prevalências de prática regular em $37 \%$ dos homens e $24 \%$ nas mulheres, diferindo pouco do que foi encontrado na pesquisa em questão. É notável que haja um decréscimo do nível de atividade física com o envelhecimento, tornando o sedentarismo um fator de risco de morbimortalidade nessa população (Matsudo e col., 2000; Siqueira e col., 2007). Diversas características encontradas na amostra, como alta prevalência de doenças crônicas, obesidade e dor, poderiam ser reduzidas substancialmente com a adoção de um estilo de vida mais ativo. Além disso, a atividade física pode ajudar os idosos a manter a independência funcional por longos períodos e pode reduzir o risco para quedas.

A atenção especial à saúde mental da população idosa deve-se particularmente pelo aumento na prevalência das demências e depressão com o avançar da idade, já que essas podem levar à perda da autonomia e anos de vida vividos com incapacidade (Lebrão e Duarte, 2003). Um estudo populacional realizado no município de São Paulo verificou, por meio do GDS, que $18,1 \%$ dos idosos apresentavam sintomas depressivos (Lebrão e Duarte, 2003), valores aproximados aos dos Grupos 1 e 2. O Grupo 3 apresentou alta prevalência de casos depressivos com 53,1\%, corroborando o estudo de Stalenhoef e colaboradores (2002) que verificaram que os sintomas depressivos estão associados às quedas recorrentes e constituem fator de risco moderado para esse evento. Esses dados mostram a influência da depressão no aumento do risco de quedas e entre seus sintomas estão: a diminuição da atenção, falta de concentração, diminuição da capacidade para assimilar e recordar informações e prolongamento do tempo de reação, que afetam diretamente o controle postural e aumentam os riscos ambientais (Nevitt, 1997). Os sintomas depressivos merecem maior atenção e esforço por parte dos profissionais da saúde na sua detecção e tratamento, pelas suas consequências debilitantes nos idosos e que em associação com as quedas podem ter efeito devastador na qualidade de vida.

A avaliação do estado cognitivo demonstrou uma piora na pontuação do MEEM conforme o histórico positivo de quedas, sendo encontrada diferença significante entre o grupo sem quedas com o de quedas recorrentes. Em estudo na área metropolitana de São Paulo, 30\% dos idosos apresentavam déficit cognitivo pelo MEEM (Ramos e col., 1998). O Grupo 1 teve valores aproximados (25\%) aos de Ramos e colaboradores (1998), mas os grupos com quedas tiveram valores superiores (Grupo 2 com 43,8\% e Grupo 3 com 53,1\%). O declínio cognitivo apresenta-se como forte fator de risco para quedas, pois leva o idoso a deteriorização das funções executivas, desorientação espacial, respostas protetoras comprometidas, comprometimento da marcha, desequilíbrio e instabilidade postural (Nevitt, 1997). Assim, o déficit cognitivo é um fator de forte associação com as quedas, não diferindo do resultado encontrado no presente estudo.

O medo de quedas foi descrito pela maioria dos idosos (59,7\%), contudo houve um aumento sem significância conforme a presença do evento quedas nos grupos, corroborando a literatura (Fabrício e col., 2004; Morris e col., 2004). Fabrício e colaboradores (2004), relataram que, entre idosos que sofreram quedas, o medo de voltar a cair foi apontado como a segunda maior consequência. Para Morris e colaborades (2004), os idosos com quedas recorrentes relataram mais medo de cair do que os que caíram uma única vez ( $p=0,0035$ ). 0 relato de quase quedas foi mais frequente nos caidores, apresentando diferença $(p=0,003)$ em comparação com os idosos sem quedas, o que revela uma autopercepção de instabilidade postural coerente com o histórico de quedas vivenciado por esses idosos. Tanto o medo de quedas como o relato de quase quedas estão associados à restrição nas atividades, pois por meio destes há a intenção de impedir que ocorra um novo evento. Fica 
claro dessa forma porque esses sintomas aumentam conforme o histórico de quedas.

Quanto ao TUGT, observou-se que os idosos do grupo sem quedas realizaram a tarefa em menor tempo, mostrando que idosos caidores apresentam maior déficit na mobilidade. Shumway-Cook e colaboradores (2000) verificaram que idosos com mais de 14 segundos no TUGT tinham um alto risco de quedas e Chiu e colaboradores (2003) ao compararem idosos que haviam caído uma única vez e seus controles, constataram diferenças significativas no desempenho do teste $(\mathrm{p}=0,025)$, resultados também encontrados nesta pesquisa.

Neste estudo, verificou-se a existência de uma série de fatores (prevalência de doenças crônicas, déficits psicocognitivos, sedentarismo, dor, tontura) que interferem na capacidade funcional, o que pode ter contribuído para um pior resultado no instrumento BOMFAQ nos idosos que sofreram quedas.

No estudo de Ramos e colaboradores (1998) com idosos da comunidade houve prevalência de dificuldade em uma a três atividades do BOMFAQ (33,7\%), com média semelhante ao da amostra total deste estudo. Em idosos com quedas recorrentes, a chance de cair aumenta em 2,37 vezes naqueles com comprometimento em uma a três atividades do BOMFAQ (Perracini e Ramos, 2002). Essa relação de piora ao $B O M F A Q$ e quedas, corrobora os resultados da presente pesquisa em que os grupos com quedas apresentaram maior dificuldade com diferença significativa em comparação ao grupo sem quedas.

As quedas são marcadores de fragilidade e da perda de capacidade funcional, não podendo ser negligenciadas. $\mathrm{O}$ idoso deve ser avaliado criteriosamente para que sejam identificados os principais fatores de risco para ocorrência de potenciais eventos no caso de idosos sem histórico de quedas e novos eventos para os caidores, com o objetivo de minimizar, por meio da prevenção, as chances de cair.

A identificação dos fatores associados ao histórico de quedas em idosos da comunidade é de grande importância para que se possam traçar, na assistência à básica saúde, métodos preventivos e de intervenção terapêutica, que devem ter o objetivo de manter ou melhorar a capacidade funcional e prevenir danos físicos, internações hospitalares e institucionalizações, diminuindo, assim, os altos custos que as quedas acarretam ao sistema de saúde e mantendo uma boa qualidade de vida para essa população.

\section{Referências}

BOULGARIDES, L. K et al. Use of clinical and impairment- based tests to predict falls by community-dwelling older adults. Physical Therapy, Alexandria, VA v. 83, n. 4, p. 328-339, april. 2003 .

BRASIL. Ministério da Saúde. Atenção básica e a saúde da família. Disponível em: <http:// http://200.214.130.35/dab/cgab/>. Acesso em: 14 abril 2008.

BROCHET, B. et al. Population-based study of pain in the elderly people: a descriptive survey. Age and Ageing, Bristol, UK., v. 27, n. 3, p. 279-289, may 1998.

CHANDLER, J. M. Equilíbrio e Quedas no Idoso: questões sobre a avaliação e o tratamento. In: GUCCIONE, A. A. Fisioterapia Geriátrica. Rio de Janeiro: Guanabara Koogan: 2002. p. 265-77.

CHIU, A. Y. Y.; AU-YEUNG, S. S. Y.; LO, S. K. A comparison of four functional tests in discriminating fallers from non-fallers in older people. Disability and Rehabilitation, Washington, DC. v. 25, n. 1, p. 45-5o, jan. 2003.

COIMBRA, A. M. et al. Falls in the elderly of the Family Health Program. Archives of Gerontology and Geriatrics, Maryland Heigts, v. 51, n. 3, p. 317322, nov.-dec. 2010.

FABRÍCIO, S. C. C.; RODRIGUES, R. A. P.; COSTA JÚNIOR, M. L. Causas e conseqüências de quedas de idosos atendidos em hospital público. Revista de Saúde Pública, São Paulo v. 38, n. 1, p. 17-24, fev. 2004 .

GAWRYSZEWSKI, V. P.; JORGE, M.H. P. M.; KOIZUMI, M. S. Mortes e internações por causas externas entre os idosos no Brasil: o desafio de integrar a saúde coletiva e a atenção individual. Revista Associação Médica Brasileira, São Paulo, v. 5o, n. 1, p. 97-103, 2004.

HERDON, J. G. et al. Chronic medical conditions and risk of fall injury events at home in older adults. Journal of the American Geriatric Society, New York, v. 45, n. 6 p. 739-743, jun. 1997. 
LEBRÃO, M. L.; DUARTE, Y. A. O. O projeto $S A B E$ no município de São Paulo: uma abordagem inicial. Brasília: Organização Pan-Americana da Saúde; 2003. 255 p.

LIPSCHITZ, D. A. Screening for nutritional status in the elderly. Primary Care, Philadelphia, Saunders, v. 21, n. 1, p. 55-67, mar. 1994.

LOURENÇO, R. A.; VERAS, R. P. Mini-Exame do Estado Mental: características psicométricas em idosos ambulatoriais. Revista de Saúde Pública, São Paulo, v. 4o, n. 4, p.712-9, ago. 2006.

MATSUDO, S. M.; MATSUDO, V. K. R.; BARROS NETO, T. L. Impacto do envelhecimento nas variáveis antropométricas, neuromotoras e metabólicas da aptidão física. Revista Brasileira de Ciência e Movimento, São Paulo, v. 8, n. 4, p. 21-32, set. 2000.

MORRIS, M. et al. Predisposing factors for occasional and multiple falls in older Australians who live at home. The Australian Journal of Physiotherapy, St. Kilda, v. 5o, n. 3, p. 153-159, 2004.

NEVITT, M. C. Falls in the elderly: risk factors and prevention. In: MASDEU, J. C.; SUDARSKY, L.; WOLFSON, L.; (edit.). Gait disorders of aging. Falls and Therapeutic strategies. Philadelphia: Lippincott-Raven Publishers, 1997. p.13-36.

PARADELA, E. M. P.; LOURENÇO, R. A.; VERAS, R. P. Validação da Escala de Depressão Geriátrica em um ambulatório geral. Revista de Saúde Pública, São Paulo, v. 39, n. 6, p. 918-23, dez. 2005.

PERRACINI, M. R.; RAMOS, L. R. Fatores associados a quedas em uma coorte de idosos residentes no comunidade. Revista de Saúde Pública, São Paulo, v. 36, n. 6, p. 709-16, dez. 2002. PODSIADLO, D.; RICHARDSON, S. The timed "Up \& Go": a test of basic functional mobility for frail elderly persons. Journal of the American Geriatric Society, New York, v. 39, n. 2, p. 142-8, feb. 1991.

RAMOS, L.R. et al.. Two-year follow-up study of elderly residents in S. Paulo, Brazil: methodology and preliminary results. Revista de Saúde Pública, São Paulo, v. 32, n. 5, p. 397-407, out. 1998.
RAMOS, L. R. et al.. Significance and management of disability among urban elderly residents in Brazil. Journal os Cross Cultural Gerontology, Dordrecht, v.8, n. 4, p. 313-23, 1993.

REKENEIRE, N. et al. Is a fall just a fall: correlates of falling in healthy older persons. The health, aging and body composition study. Journal of the American Geriatric Society, New York, v. 51, n. 6, p. 841-846, june 2003.

ROMANI, F. A. Prevalência de transtornos oculars na população de idosos residentes na cidade de Veranópolis, RS, Brasil. Arquivo Brasileiros de Oftalmologia, São Paulo, v. 68, n. 5, p. 649-655, out. 2005 .

SHUMWAY-COOK, A.; BRAUER, S.; WOOLLACOTT, $M$. Predicting the probability for falls in community-dwelling older adults using the Timed Up \& Go test. Physical Therapy, Alexandria, VA, v. 8o, n. 9, p. 896-903, sept. 2000.

SIQUEIRA, F. V. et al. Prevalência de quedas em idosos e fatores associados. Revista de Saúde Pública, São Paulo, v. 41, n. 5, p. 49-56, out. 2007.

STALENHOEF, P.A. et al. A risk model for the prediction of recurrent falls in communitydwelling elderly: a prospective cohort study. Journal of Clinical Epidemiology, v. 55, n. 11, p. 1088-1094, nov. 2002.

TINETTI, M. E.; WILLIAMS, C. S.; GILL, T. M. Health, functional and psychological outcomes among older persons with chronic dizziness. Journal of the American Geriatric Society, New York, v. 48, n. 4, p. 417-21, april 2000.

WORLD HEALTH ORGANIZATION. International Classification of Diseases. $9^{\text {th }}$ Revision (ICD-9). Geneva: WHO, 1977.

YUSUF, H. R. et al. Leisure-time physical activity among older adults: United States, 1990. Archives of Internal Medicine, Chicago, v. 156, n. 12, p. 13216, june 1996.

Recebido em: 20/04/2009

Reapresentado em: 03/09/2010

Aprovado em: 21/09/2010 